
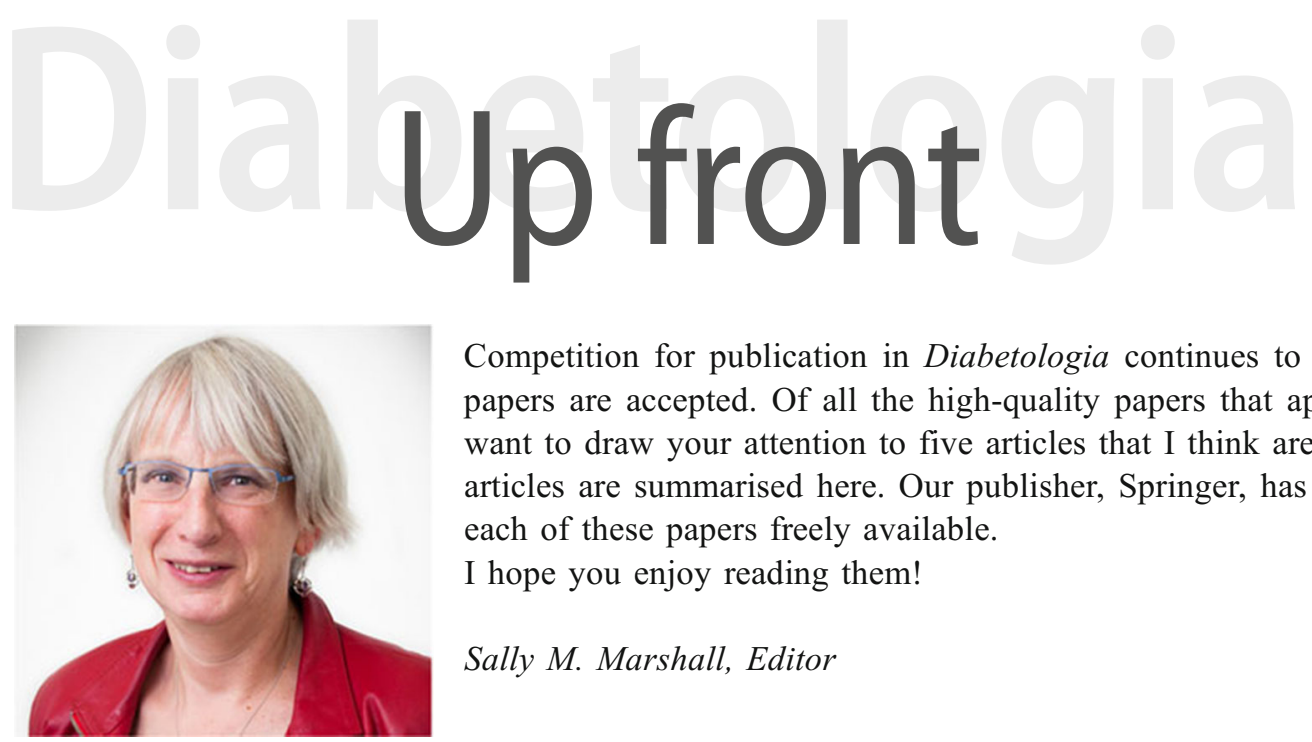

Competition for publication in Diabetologia continues to grow, and less than $20 \%$ of papers are accepted. Of all the high-quality papers that appear in this month's issue I want to draw your attention to five articles that I think are particularly interesting. The articles are summarised here. Our publisher, Springer, has kindly made the full text of each of these papers freely available.

I hope you enjoy reading them!

Sally M. Marshall, Editor

\section{Genomic annotation of disease-associated variants reveals shared functional contexts}

Yasuhiro Kyono, Jacob O. Kitzman, Stephen C. J. Parker

Genome-wide association studies (GWAS) of diabetes and related glycaemic traits have collectively identified hundreds of independent risk-associated single nucleotide polymorphisms (SNPs). The majority of these loci (>90\%) localise to non-coding regions of the genome, strongly suggesting gene regulation as a major driving mechanism. In this issue, Kyono et al (https://doi.org/10.1007/s00125-019-48233 ) review how the generation and analysis of epigenomics datasets have enabled insights into the biology that underlies these regions of genetic predisposition to disease. They review existing and emerging tools that can be used to determine which subset of SNPs might be functionally significant and the context in which their function may emerge. Collectively, recent literature suggests that different diabetes and related trait SNPs scattered throughout the genome may operate through the same transcriptional regulatory pathways.

(1) The figures from this review are available as a downloadable slideset.
Spontaneous ketonuria and risk of incident diabetes: a 12 year prospective study

Gyuri Kim, Sang-Guk Lee, Byung-Wan Lee, Eun Seok Kang, Bong-Soo Cha, Ele Ferrannini, Yong-ho Lee, Nam H. Cho

Ketones are regarded as a thrifty fuel for peripheral tissues, but the clinical and prognostic significance of mild ketosis is still uncertain. In this issue, Kim and colleagues (https://doi.org/10. $1007 / \mathrm{s} 00125-019-4829-\mathrm{x}$ ) investigated the association between spontaneous fasting ketonuria and incident diabetes in conjunction with changes in metabolic variables in a large population-based, observational study. During 12 years of follow-up, individuals with fasting ketonuria at baseline maintained lower post-load $1 \mathrm{~h}$ and $2 \mathrm{~h}$ glucose levels and a higher insulinogenic index, even though the groups with and without ketonuria had comparable baseline values. Individuals with spontaneous fasting ketonuria at baseline had a significantly lower risk of incident diabetes compared with individuals without ketonuria, independently of other metabolic variables. The authors suggest that spontaneous fasting ketonuria may be a novel signature in the modulation of glucose metabolism and may have the potential to prevent diabetes. 
No evidence of a causal association of type 2 diabetes and glucose metabolism with atrial fibrillation

Hadi Harati, Daniela Zanetti, Abhiram Rao, Stefan Gustafsson, Marco Perez, Erik Ingelsson, Joshua W. Knowles

Type 2 diabetes and less marked forms of dysglycaemia have been associated with increased risk of incident atrial fibrillation in epidemiological studies. However, it is unclear whether this association is causal. In this issue, Harati et al (https://doi. org/10.1007/s00125-019-4836-y) used Mendelian randomisation to answer this question. Using publicly available summary statistics data from genome-wide association studies, the authors did not find a causal role between genetically programmed type 2 diabetes, fasting blood glucose or $\mathrm{HbA}_{1 \mathrm{c}}$ and the development of atrial fibrillation. These data suggest that drug treatment to reduce dysglycaemia is unlikely to be an effective approach for the prevention of atrial fibrillation. The authors conclude that, in line with the previously reported causal link between BMI and atrial fibrillation, these results suggest that strategies that aim to control type 2 diabetes-related comorbidities (such as obesity) are likely to be more effective in preventing atrial fibrillation than those that aim to control blood glucose.

Black African men with early type $\mathbf{2}$ diabetes have similar muscle, liver and adipose tissue insulin sensitivity to white European men despite lower visceral fat

Oluwatoyosi Bello, Cynthia Mohandas, Fariba ShojeeMoradie, Nicola Jackson, Olah Hakim, K. George M. M. Alberti, Janet L. Peacock, A. Margot Umpleby, Stephanie A. Amiel, Louise M. Goff

Black African populations experience disproportionately high rates of type 2 diabetes but typically present with less visceral fat deposition than other ethnic groups. In this issue, Bello et al (https://doi.org/10.1007/s00125-019-4820-6) use highly sensitive techniques to investigate ethnic differences in visceral fat and tissue-specific insulin sensitivity between men of black African and white European ethnicity with type 2 diabetes. They report comparable insulin sensitivity in the liver, skeletal muscle and adipose tissue in the two groups, despite lower visceral fat in the black African men. The authors suggest that excess adiposity, particularly visceral deposition, is a smaller driver of insulin resistance in black African men than in white European men and this supports the notion that there may be ethnic differences in the development of type 2 diabetes.

Selective deletion of endothelial cell calpain in mice reduces diabetic cardiomyopathy by improving angiogenesis

Xiaomei Teng, Chen Ji, Huiting Zhong, Dong Zheng, Rui Ni, David J. Hill, Sidong Xiong, Guo-Chang Fan, Peter A. Greer, Zhenya Shen, Tianqing Peng

It is well known that cardiomyocyte dysfunction plays a central role in diabetic cardiomyopathy. However, the role of non-cardiomyocytes in this diabetic complication has not been fully addressed. In this issue, Teng et al (https://doi.org/10.1007/s00125-019-4828-y) report that endothelial cell-specific disruption of calpain reduces cardiomyopathy in a mouse model of diabetes and that the protective effect of endothelial cell-specific calpain disruption may be attributed to attenuated endothelial injury and improved angiogenesis in diabetic hearts. The authors suggest that endothelial cell calpain may promote endothelial cell death and inhibit neovascularisation via suppression of $\beta$-catenin in diabetes. They conclude that calpain-mediated endothelial cell dysfunction may be important in the pathogenesis of diabetic cardiac complications and, thus, pharmaceutical approaches targeting calpain may prove useful for the treatment of diabetes and its cardiovascular complications.

All text supplied by the authors.

Publisher's note Springer Nature remains neutral with regard to jurisdictional claims in published maps and institutional affiliations. 